Document downloaded from:

http://hdl.handle.net/10251/78689

This paper must be cited as:

Molina, M.; Lopez, C.; Staltari, S.; Chorzempa, S.; Moreno Ferrero, V. (2013). Cryptic homoelogy analysis in species and hybrids of genus Zea. Biologia Plantarum. 57(3):449456. doi:10.1007/s10535-012-0299-4.

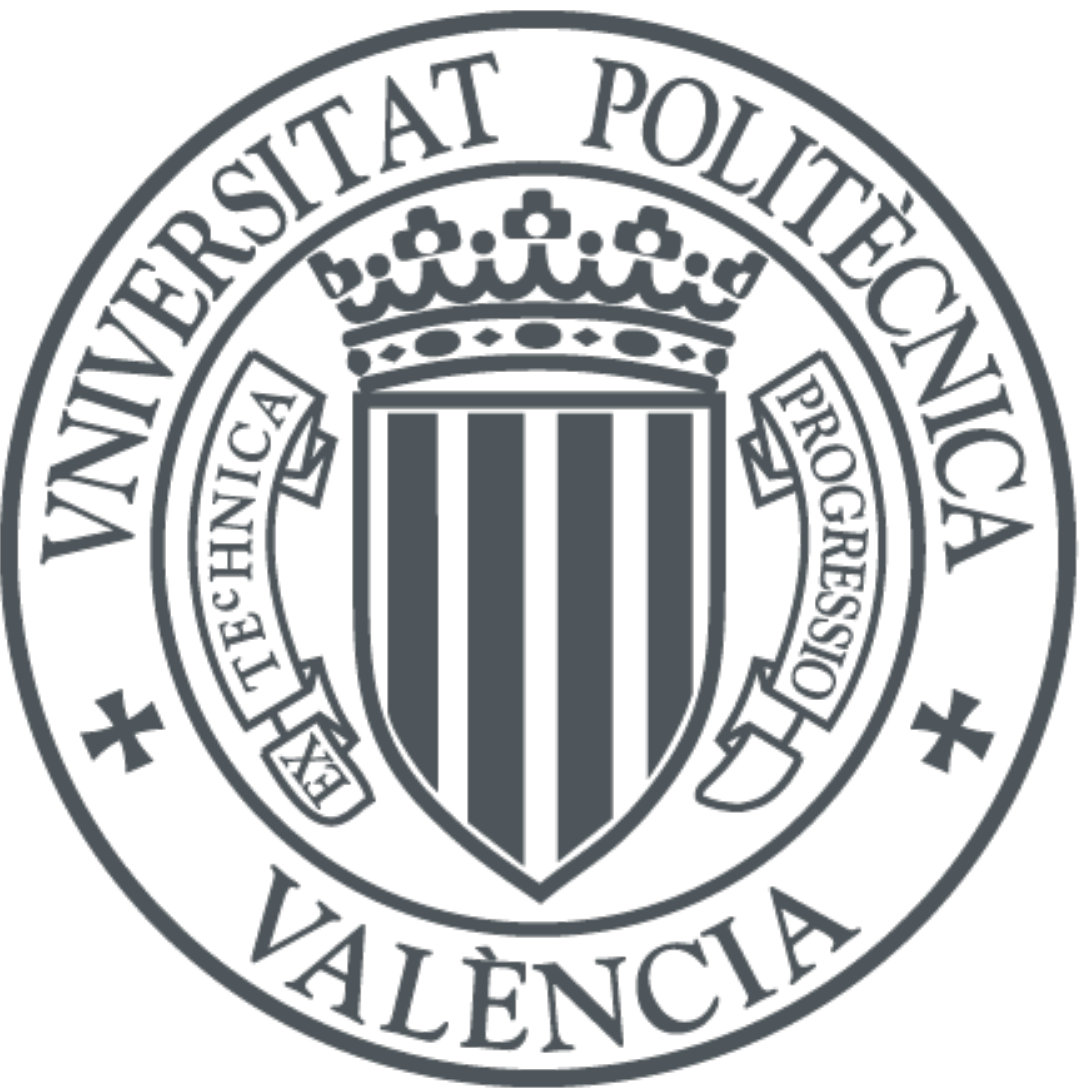

The final publication is available at

http://dx.doi.org/ 10.1007/s10535-012-0299-4

Copyright Springer Verlag (Germany)

Additional Information 


\title{
Cryptic homoeology analysis in species and hybrids of genus Zea
}

\section{DEL C. MOLINA ${ }^{1,2 *}$, C.G. LÓPEZ ${ }^{3}$, S. STALTARI ${ }^{1}$, S.E. CHORZEMPA ${ }^{3}$, V. MORENO FERRERO 4}

${ }^{1}$ Instituto Fitotécnico de Santa Catalina, Facultad de Ciencias Agrarias y Forestales, Universidad Nacional de la Plata. Garibaldi 3400. Llavallol, Buenos Aires, Argentina. TE-FAX (+54) 114282 0233. momgen@yahoo.com

${ }^{2}$ Consejo Nacional de investigaciones Científicas y Técnicas (CONICET). Rivadavia 1917, Ciudad Autónoma de Buenos Aires, Argentina.

${ }^{3}$ Facultad de Ciencias Agrarias, Universidad Nacional de Lomas de Zamora, Ruta 4 Km. 2, Buenos Aires, Argentina.

${ }^{4}$ Departamento de Biotecnología, Universidad Politécnica de Valencia and Instituto de Biología Molecular y Celular de Plantas (UPV-CSIC), Valencia, Spain.

\begin{abstract}
Cryptic intergenomic pairing of genus Zea was induced by the use of a diluted colchicine solution, in order to elucidate the phylogenetic relations and differentiation of the homoeologous genomes. Results indicate that in species and hybrids with $2 n=20$, there was chromosome pairing between the homoeologous $\mathrm{A}$ and $\mathrm{B}$ genomes with a maximum of 5IV, with the exception of Zea diploperennis and their interspecific hybrids, where cryptic homoeologous chromosome pairing was not induced. In almost all $2 n=30$ hybrids, observed cryptic pairing increased to a maximum of $10 \mathrm{III}$, although $Z$. mays $x$ Z. mays with $2 n=30$, did not show significant differences between treated and untreated materials. Pairing was also observed in species and hybrids with $2 n=40$, in which a maximum of $10 \mathrm{IV}$ was observed, with
\end{abstract}


the exception of $Z$. mays with $2 n=40$ where treated and untreated cells did not differed significantly.

Additional key words: colchicine, genome, maize, subgenome, teosinte.

Abbreviations: A - subgenome A Zea; B - subgenome B Zea; Bp1 - subgenome Bp1 Zea perennis; Bp2 - subgenome Bp2 Zea perennis; 2,4-D - 2,4 dichlorophenoxyacetic; I chromosome monovalent; II - chromosome bivalent; III - chromosome trivalent; IV chromosome quadrivalent; MDL - (Zea mays x Zea diploperennis) x Zea luxurians; MDP - Zea mays ssp mays $2 \mathrm{n}=40 \times$ (Zea diploperennis $x$ Zea perennis) $2 \mathrm{n}=40$; pam1 - gene plural abnormalities of meiosis; $\mathrm{Ph}$-gene pairing homoeologous; Ph1 - pairing homoeologous 1; Zd - Zea diploperennis; ZdxZI - Zea diploperennis x Zea luxurians; ZdxZp30 - Zea diploperennis $x$ Zea perennis $2 \mathrm{n}=30$; ZdxZp40 - Zea diploperennis $x$ Zea perennis $2 \mathrm{n}=40$; Zl - Zea luxurians; ZlxZp30 - Zea luxurians $x$ Zea perennis $(2 \mathrm{n}=30) ; \mathrm{Zm}$ - Zea mays ssp mays $2 \mathrm{n}=$ 20; Zm40 - Zea mays ssp mays 2n = 40; Zmex - Zea mays ssp mexicana; ZmxZd - Zea mays ssp mays x Zea diploperennis ; ZmxZl - Zea mays ssp mays $x$ Zea luxurians; ZmxZm30 Zea mays ssp mays $(2 \mathrm{n}=40)$ x Zea mays ssp mays $(2 \mathrm{n}=20) ; Z m x Z m e x-Z e a$ mays ssp mays $x$ Zea mays ssp mexicana; ZmxZp30 - Zea mays ssp mays $x$ Zea perennis $2 \mathrm{n}=30$; ZmxZp40 - Zea mays 2n = $40 \times$ Zea perennis; ZmxZpar - Zea mays ssp mays $x$ Zea mays ssp parviglumis; Zp - Zea perennis; Zpar - Zea mays ssp parviglumis; ZpxZmex - Zea perennis $x$ Zea mexicana; ZparxZd40 - Zea mays ssp parviglumis $x$ Zea diploperennis $2 \mathrm{n}=$ 40.

\section{ACKNOWEDGMENTS}

This research was supported by the Universidad Nacional de la Plata, Universidad Nacional de Lomas de Zamora and CONICET. 


\section{Introduction}

Zea is an important genus of the tribe Maydeae. According to Doebley and Iltis (1980), Iltis and Doebley (1980) and Iltis and Benz (2000) it is composed of two section: Section Luxuriante, which includes the annuals teosintes Zea luxurians and Zea nicaraguensis and the perennials Zea diploperennis and Zea perennis and Section Zea comprises only an annual species (Z. mays $\mathrm{L}$ ) which can be divided into three subspecies: $Z$. mays ssp. mays (maize) and the teosintes Z. mays ssp. mexicana and Z. mays ssp. parviglumis. All the species mentioned above have $2 n=20$ chromosome except $Z$. perennis which has $2 n=40$.

The maize genome is characterized by having a large number of duplicated genes (Wendel 2000). Three models can explain the large-scale duplications in the maize genome, that is, segmental duplication (multiple independent duplications within a genome), autotetraploidy (intraspecific genomic duplication), and allotetraploidy (interspecific genome hybridization).

Swigonová et al. 2004, support a theory in which maize has a tetraploid origin. This analysis also indicates a contemporaneous divergence of the ancestral sorghum genome and the two maize progenitor genomes about 11.9 million years ago (Mya). On the basis of a putative conversion event detected for one of the genes, tetraploidization must have occurred before 4.8 Mya, and therefore, the major maize genome expansion.

According to Schnable et al. 2011 and Schnable and Freeling 2011, maize is a tetraploid species with two differentiated parental genomes, maize1 and maize2. Maize1 is the subgenome that experienced less gene losses followed by the whole genome duplication in maize lineage 5-12 Mya. Genes located on this subgenome tend to be expressed at a higher level in modern maize (Schnable and Freeling 2011). 
Colchicine concentrations of $0.5 \times 10^{-4} \mathrm{M}$ induce homoeologous chromosome pairing (Poggio et al. 1990, Molina 2011), with the formation of heteromorphic bivalents or multivalents (Driscoll et al 1967, Feldman and Avivi 1988), which favors intergenomic pairing in species with homoeologous genomes (Dover and Riley 1973).

Driscoll and Darvey (1970) observed that colchicine affects the spatial relationship of homoeologous chromosomes but not the formation of chiasmata. Additionally, they suggested that chromosome position is crucial in the meiotic pairing of homologous and homoeologous chromosomes, altering the arrangement of chromosomes in the nuclear membrane, and allowing the expression of cryptic genomic homology.

Jackson (1982) described a model that explains the chromosome pairing and chiasma formation in homologous and homoeologous genomes, in which there is a genetic control of the specific binding site of chromosomes to the nuclear membrane, under the regulation of the Ph gene. Jackson and Murray (1983) demonstrated that the application of diluted colchicine solution to meiotic cells can break the genetic control of these genes, promoting the intergenomic pairing, and thus revealing the cryptic homology in polyploids.

In maize, the pam1 gene (plural abnormalities of meiosis 1) is associated with the formation of the bouquet by intervening in the telomere anchoring to the nuclear membrane, and by facilitating homologous chromosome pairing. Golubovskaya et al. (2002) concluded that the pam1 gene plays an important role in the formation of the bouquet and in the pairing of homologous chromosomes.

Several researchers have treated premeiotic cells of Zea mays, Z. perennis, Z. diploperennis and their hybrids with diluted colchicine solutions (Poggio et al. 1990, Molina and García 1999, 2000, 2001, Molina et al. 2004, 2005, Molina 2011, González and Poggio 2011). Up to 5 quadrivalents have been observed in maize and hybrids with $2 \mathrm{n}=20$, while a higher number 
of quadrivalents has been found in Zea perennis when compared to untreated controls. Colchicine treatment has been observed to favor homoeologous chromosome pairing, and more than one mechanism has been proposed to explain such effect, modification of the position of the chromosomes in the nuclear membrane or alteration in the formation of the bouquet (Bass et al. 2000), and annulment of the expression of a maize gene, equivalent to the Phgene of wheat (Poggio et al. 1990, Molina et al. 2004).

In the present study, cryptic homology in species and hybrids of the genus Zea was analyzed, with the aim to explore the phylogenetic relationship among Zea species, by using diluted colchicine solutions that induce intergenomic chromosome pairing.

\section{MATERIALS AND METHODS}

\section{MATERIALS}

\section{PARENTAL SPECIES}

\section{Section Zea}

Zea mays ssp mays $2 n=20(Z m)$ : Open pollinated population Colorado Klein. Inbred line knobless, from the Maize Genetic Cooperation Stock Center (MGCSC), Urbana, Illinois, USA.

Zea mays ssp mays 2n = 40 (Zm40). Sugary inbred lines N103A, N104B, N107C, N107B, and 90-2189-2190, from MGCSC.

Zea mays ssp mexicana 2n = 20 (Zmex). From CIMMYT (Mexico).

Zea mays ssp parviglumis $\mathbf{2 n}=\mathbf{2 0}$ (Zpar). From Dr. Bird, CIMMYT (Mexico).

\section{Section Luxuriantes}

Zea luxurians 2n = 20 (ZI). From Guadalajara, Mexico.

Zea diploperennis 2n = $\mathbf{2 0}$ (Zd). From Sierras Occidentales de Manjatlan, Jalisco, Mexico. Courtesy of Dr. Iltis.

Zea perennis 2n = 40 (Zp). From Ciudad Guzmán, Jalisco, Mexico. Courtesy of Dr. Prywed, introduced in 1962 at the Instituto Fitotécnico de Santa Catalina, Llavallol, Argentina. 


\section{HYBRIDS}

\section{Dihybrids}

$2 n=20$

Zea mays ssp mays $\mathrm{x}$ Zea mays ssp mexicana (ZmxZmex).

Zea mays ssp mays $\mathrm{x}$ Zea mays ssp parviglumis (ZmxZpar).

Zea mays ssp mays $x$ Zea luxurians (ZmxZl).

Zea mays ssp mays $x$ Zea diploperennis (ZmxZd).

Zea diploperennis x Zea luxurians (ZdxZI).

$2 n=30$

Zea mays ssp mays $(2 \mathrm{n}=40) \times$ Zea mays ssp mays $(2 \mathrm{n}=20),(Z m \times Z m 30)$.

Zea mays ssp mays $(2 \mathrm{n}=40) \times$ Zea parviglumis $(Z \mathrm{~m} \times Z$ par30).

Zea mays ssp mays $x$ Zea perennis (ZmxZp30).

Zea diploperennis $x$ Zea perennis (ZdxZp30).

Zea luxurians x Zea perennis (ZlxZp30).

Zea perennis $x$ Zea mexicana (ZpxZmex).

$2 n=40$

Zea mays with $2 \mathrm{n}=40 \times$ Zea perennis $(\mathrm{Zm} \times \mathrm{Zp} 40)$.

Zea mays ssp parviglumis $x$ Zea diploperennis $2 n=40$, obtained by chromosome duplication of the $2 n=20$ hybrid (ZparxZd40).

Zea diploperennis $x$ Zea perennis, obtained by crossing a non-reduced gamete of Zea diploperennis with a normal gamete of Zea perennis (ZdxZp40).

\section{Trihybrids}

$2 n=20$

A cross between hybrid (Zea mays $x$ Zea diploperennis) x Zea luxurians (MDL). 
$2 n=40$

Zea mays ssp mays $2 \mathrm{n}=40 \times$ (Zea diploperennis $x$ Zea perennis) $2 \mathrm{n}=40$ (MDP).

\section{METHODS}

\section{Field experiments}

Crosses and self pollinations of maize inbred lines and Teosinte populations were made at field and greenhouse conditions. When Teosinte was used as male, before pollination, maize silks were cut to 3 or $4 \mathrm{~cm}$, since the maximum length of the pollinic tube in Teosinte is about 6 to $7 \mathrm{~cm}$, unlike maize, whose pollinic tube can reach more than $30 \mathrm{~cm}$. In crosses between species whose hybrids have a chromosome number of $2 n=30$, which are generally difficult to obtain due to a limited embryo growth, a solution of $0.45 \mathrm{mM}$ of 2,4 dichlorophenoxyacetic (2,4-D) (Furini and Jewel 1995) was applied to the ears, two days after pollination, in order to favor embryo development.

\section{Rescue and embryo culture}

Immature embryos from $2 n=30$ hybrids were rescued and cultured in vitro. About 12 to 30 days after fecundation, the embryo collapsed and died. The survival period depended on the environmental conditions. If the plants are grown under optimal field conditions for maize development, embryos can survive for 12 days, whereas under greenhouse conditions during winter, the development of embryos is generally delayed and survival is longer (21 to 30 days).

Ears were harvested when embryos had a maximum length of $1 \mathrm{~mm}$. Caryopses were washed with a $2.5 \%$ sodium hypochlorite solution and then sown in a basic medium (García and Molina 1992, 2001) supplemented with $4.5 \mu \mathrm{mol} \mathrm{dm}^{-3}$ de 2,4-D. Treated caryopses were incubated in a growth chamber with a photoperiod of $16 \mathrm{~h}$ light and $8 \mathrm{~h}$ of darkness, subcultured every 30 days in a callus maintenance medium (García and Molina 1992). 
Regenerated plants were transplanted into a culture medium free of 2,4-D to develop roots. Subsequently, plants were transplanted and acclimated in a greenhouse.

\section{Treatment with diluted colchicine solution}

Immature tassels from different Zea species and hybrids were cut and introduced in a $0.5 \mathrm{x}$ $10^{-4} \mathrm{M}$ colchicine solution for $12 \mathrm{~h}$, and then stored in distilled water for $24 \mathrm{~h}$ (controls were stored for $36 \mathrm{~h}$ in distilled water). Then, the treated material and the controls were fixed in Farmer solution (3:1 ethylic alcohol/acetic acid), where they were conserved for 8 to 10 days, and then stored at $4{ }^{\circ} \mathrm{C}$ in an alcohol 70 solution.

\section{Cytogenetic analysis}

To analyze the meiotic configurations in parental species and hybrids, anthers excised from male florets previously fixed in Farmer solution were squashed in a drop of $2 \%$ ferric hematoxylin, using a micro-drop of ferric acid as mordant. Between 130 and 224 cells were analyzed in controls and treatments.

Significant differences between meiotic configurations (control vs. the corresponding treatment) were tested using the Mann-Whitney $U$ test (Sokal and Rohlf 1978) at a 5\% probability level (STATISTICA program version 7).

\section{Chromosomal notation}

I (monovalent); II (bivalent); III (trivalent); IV (quadrivalent)

\section{Results}

\section{Effect of the colchicine diluted solution on the cryptic pairing of the Zea complex}

To obtain the cryptic pairing of homoeologous chromosomes of different genomes from species and hybrids of the Zea complex, tassels were treated with a diluted colchicine solution, with the following results: 
In Zea species with $2 \mathrm{n}=20$ :

In Z. mays with $2 \mathrm{n}=20$ (Table $1 \mathrm{~A}), Z$. mexicana (Table 1B), Z. parviglumis (Table 1C) and $Z$. luxurians (Table 1D), colchicine induced the homoeologous chromosome pairing of the two relict genomes, designated as genomes $A$ and $B$ (Fig. 2), with a maximum of $5 \mathrm{IV}$ (Table 1A, 1B, 1C, 1D and Fig. 1A). The exception was $Z$. diploperennis (Table 1E), in which no quadrivalents formation was observed using the colchicine concentration that showed quadrivalents in the other species.

In Zea hybrids with $2 \mathrm{n}=20$ :

Cryptic pairing was observed in Z. mays $x$ Z. mexicana (Table 2A), Z. mays $x$ Z. parviglumis with $2 \mathrm{n}=20$ (Table $2 \mathrm{~B}), Z$. mays $\times$ Z. luxurians (Table $2 \mathrm{C}$ ) and the trihybrid (Z. mays $\times Z$. diploperennis) $x$ Z. luxurians (Table $2 \mathrm{~F}$ ), although in different proportions depending on the hybrid analyzed. The highest percentage of pairing was observed in Z. mays $\times$ Z. parviglumis with $2 \mathrm{n}=20$ (Table 2B). On the other hand, cryptic pairing of homoeologous chromosomes of the A and B genomes was not observed in Z. mays $x$ Z. diploperennis with $2 \mathrm{n}=20$ (Table 2D) and Z. diploperennis $\times$ Z. luxurians hybrids (Table 2E). In both cases, one of the parents was Z. diploperennis, which also showed no induction of homoeologous chromosome pairing (Table 1E).

In Zea hybrids with $2 \mathrm{n}=30$ :

No significant differences between treated material and controls were observed in the hybrid between Zea mays with different ploidy levels (Table 3A). The most frequent configuration was $8 I I I+2 I I+2 I$. In the hybrids $Z$. mays $x Z$. parviglumis with $2 \mathrm{n}=30$ (Table $3 \mathrm{~B}$ ) and Z. luxurians $x$ Z. perennis with $2 \mathrm{n}=30$ (Table $3 \mathrm{E}$ ), cryptic pairing was increased, with a higher percentage of trivalents in the treated material, in comparison with the controls. A increase in cryptic pairing was also observed in Z. perennis $x$ Z. mexicana (Table 3D) and Z. diploperennis $x$ Z. perennis 
with $2 n=30$ (Table 3F). While treated material showed up to $10 \mathrm{III}$, a maximum of 6III were observed in the controls (Table 3F). The highest difference between treated genotypes and controls was observed in the $2 n=30$ hybrid $Z$. mays $x Z$. perennis (Table $3 C$ ), where the meiotic configuration most frequently observed was $5 I I I+5 I I+5 I$ for controls and $8 I I I+2 I I+2 I$ for the treated hybrids.

In species and hybrids with $2 \mathrm{n}=40$ :

In the $2 n=40$ maize, only a slight increase in the meiotic configuration of 9IV+2II or $10 \mathrm{IV}$, and a minimal decrease in the configurations with less than 7IV was observed (Table 4A). The most frequent meiotic configuration in $Z$. perennis controls was $5 \mathrm{IV}+10 \mathrm{II}$, and less frequently $6 \mathrm{IV}+8 \mathrm{II}$. In the treated material, the percentage of quadrivalents increased to a maximum of 10 , with an average of $7 \mathrm{IV}+6 \mathrm{II}$ (Table $4 \mathrm{~B})$.

In Z. mays $x$ Z. perennis hybrids with $2 n=40$ (Table $4 \mathrm{C}$ and Fig. 1D), Z. diploperennis $\times$ Z. perennis with $2 \mathrm{n}=40$ (Table 4D) and the trihybrid Z. mays $x$ (Z. diploperennis $x$ Z. perennis) (Table 4F), there was a increase in the number of IV treated material, reaching a maximum of 10IV. In all cases, controls showed a very low proportion of pairing among homoeologous chromosomes, especially between $Z$. diploperennis and Z. perennis (Table 4D).

The behavior of the hybrid Z. parviglumis $x Z$. diploperennis with $2 n=40$, obtained by chromosome duplication of $Z$. parviglumis $x Z$. diploperennis with $2 \mathrm{n}=20$, was different from that of the rest of the hybrids with the same chromosome number (Table 4E). The most frequent meiotic configuration in the control was $2 \mathrm{IV}+16 \mathrm{II}$. Apparently, pairing of identical chromosomes in this hybrid was favored, reducing the homoeologous pairing between the $\mathrm{A}$ and B genomes, which could explain the low frequency of IV observed. The treated material showed results similar to the rest of the hybrids studied (Table 4E). 
In all the cases, the number of chiasma increased in the treated material (Table 5), except in Z. diploperennis, maize with $2 n=40$, the hybrid $Z$. mays $x Z$. mays $2 n=30, Z$. mays $x Z$. diploperennis, and Z. diploperennis $x$ Z. luxurians with $2 \mathrm{n}=20$ (Table 5).

\section{Discussion}

\section{Cryptic pairing among homoeologous chromosomes of the genus Zea}

The analysis of the meiotic behavior of Zea species with $2 n=20$ after treatment with a diluted colchicine solution (Table 1A, 1B, 1C and 1D) showed homoeologous chromosome pairing in all species (with a maximum of 5IV), with the exception of Zea diploperennis, which did not show significant differences when compared to the treated and controls (Table 1E).

More than one possible reason can be mentioned to explain the lack of pairing in Zea diploperennis. First, the expected alteration of the spatial relation of chromosomes (Driscoll and Darvey 1970) was not observed, hence, they were able to maintain their ordering (Feldman and Avivi 1988, Feldman et al. 1997) and anchor to the nuclear membrane (Bass et al. 2000, Chikashige et al. 2010), favoring pairing among homologous chromosomes. Second, the pam1 gene, which intervenes in the presynaptic mechanisms (Felman and Avivi 1988, Bozza and Pawlowski 2008) and in the chromosome reorganization related to the formation of the bouquet (Zickler and Kleckner 1998, Bass et al. 2000), may have a different effect on $Z$. diploperennis than on the other Zea species. Third, the action of an equivalent $P h$ gene in $Z$. diploperennis is not inhibited by the diluted colchicine solution used. Fourth, Z. diploperennis chromosomes are not homoeologous, which is possible if $Z$. diploperennis was obtained from a haploid Z. perennis. Independently of which is the mechanism that resulted in the lack of pairing observed, it would be interesting to analyze the last hypothesis in future researches.

Cryptic pairing of homoeologous chromosomes in $2 n=20$ hybrids of the genus Zea (Table 2) varied according to the cross analyzed, but was higher in the hybrids between more closely 
related species (Z. mays, Z. mexicana and $Z$. parviglumis) than in more distant species $(Z$. luxurians and Z. diploperennis).

The hybrids $Z$. mays $x$ Z. luxurians (Table $2 \mathrm{C}$ ) and (Z. mays $x Z$. diploperennis) $x$ Z. luxurians (Table 2F) showed a similar cryptic pairing, but resulted lower than in species with higher chromosome affinity. The cryptic pairing observed in the hybrid Z. mays $x$ Z. luxurians (Table 2C) maybe attributable to a higher differentiation among their homoeologous chromosomes, since parental species are evolutionarily more distant.

The meiotic behavior of Zea in the species and hybrids with $2 n=30$ after treatment with colchicine showed no significant differences between treated and untreated material in the hybrids between species of $Z$. mays (Table $3 \mathrm{~A}$ ). A possible explanation is the fact that this hybrid is a cross between genotypes of the same species with different ploidy level, and the preferential pairing is between homologous chromosomes. The preferential pairing of homologous chromosomes was observed by Santos and Orellana 1983, Santos et al. 1984, Jenkinsy and Chatterjee 1994, Jenczewski and Alix 2004. However, it is not clear why $2 n=20$ maize showed cryptic pairing between the A and B genomes (Table $1 A$ and Fig. 1A), which was inhibited with a higher ploidy level (Table 4A).

A high level of homoeologous chromosome pairing, with a maximum of $10 \mathrm{lll}$, was detected in untreated Z. mays $\times$ Z. parviglumis with $2 \mathrm{n}=30$ (Table 3B) and Z. luxurians $\times$ Z. perennis (Table 3E). Colchicine increased the percentage of III only slightly.

The highest response to colchicine treatment, with a maximum of $10 \mathrm{III}$, was obtained in the $2 \mathrm{n}$ $=30$ hybrid $Z$. mays $\times Z$. perennis (Table 3C and Fig. $1 \mathrm{C}$ ), suggesting that chromosomes of $Z$. mays and Z. perennis are homoeologous, even though both species are evolutionarily differentiated. 
Different results were found in $2 n=40$ species and hybrids. Treated and untreated $Z$. mays showed pairing between $A$ and $B$ homologous chromosomes, with a maximum of 10IV (Table 4A). Colchicine treatment did not induce homoeologous pairing and no hexavalents or octovalents chromosomes were observed (Table 4A).

Treatment in Z. perennis induced only pairing of homoeologous chromosomes of the $B$ genome (Table 4B and Fig. 1B), with a maximum of 10IV. Genome B chromosomes paired as bivalents, while genome A chromosomes paired as quadrivalents. There was no pairing between chromosomes of the $\mathrm{A}$ and $\mathrm{B}$ genome.

Considering Zea mays with $2 n=40$ (Table 4A), Z. diploperennis $x$ Z. perennis $2 n=40$ (Table 4D), Z. mays $\times$ Z. perennis $2 \mathrm{n}=40$ (Table 4C and Fig.1D) and $Z$, mays $x$ (Z. diploperennis $x Z$. perennis) (Table 4F), chromosomes of the A genome from species that form the hybrids paired similarly in both treated and untreated material. Colchicine induced the cryptic pairing of homologous chromosomes of the $\mathrm{B}$ genome, with a maximum of $10 \mathrm{IV}$.

\section{Possible evolution of the genomes of Zea}

Previous results (Molina and Naranjo 1987, Molina and García 2001, Molina et al. 2004, Molina 2011, Poggio et al. 1990, Naranjo et al. 1989, 2004, González and Poggio 2011) support the theory that consider Zea as an autotetraploid with two subgenome with different level of conservation (Swigonová et al. 2004, Schnable and Freeling 2011, Schnable et al. 2011).

A possible mechanism of differentiation of subgenomes which could have led to different Zea species would consist in a duplication of the A genome of a diploid species, followed by a differentiation in the homoeologous genomes A and B (Fig. 2). Another possible mechanism that resulted in the genotypic constitution of $A A B B$ individuals is an ancestral cross between two diploid species (AA and $B B)$. Our results support the theory of a chromosome duplication 
followed by a differentiation of two genomes, since in the hybrid Zea mays ssp. parviglumis $\mathrm{x}$ Zea diploperennis with $2 n=40$ (obtained by crossing two Zea species and then duplicated) chromosomes of each species paired preferentially with the homologous instead of homoeologous chromosomes, resulting in a more frequent configuration of $2 \mathrm{IV}+16 \mathrm{II}$, while in most hybrids the configuration was $5 \mathrm{IV}+10 \mathrm{Il}$. The $\mathrm{A}$ genome would have remained undifferentiated, allowing the current pairing of homoeologous chromosomes of interspecific hybrids as if they were homologous. The differentiation of B genomes would be the evolutionary process ((Fig. 2) which would have resulted in different species of Zea, probably favoring their geographic isolation (Ruiz et al. 2001, Fukunaga et al. 2005) or domestication (Swanson-Wagner et al. 2012)

\section{REFERENCES}

Bass, H.W., Riera-Lizarazu, O., Ananiev, E.V.B., Bordolini, S.J., Rines, H.W., Phillips, R.L., Sedat, J.W., Agard, D.A., Cande, Z.W.: Evidence for the coincident initiation of homologous pairing and synapsis during the telomere-clustering (bouquet) stage of meiotic prophase. - J. of Cell Sci. 113: 1033-1042, 2000.

Bozza, C.G., Pawlowsky, W.P.: The cytogenetics of homologous chromosome pairing in meiosis in plants. - Cytogenet. Gen. Res. 120: 313-319, 2008.

Chikashige, Y., Haraguchi, T., Hiraoka, Y.: Nuclear envelope attachment is not necessary for telomere function in fission yeast. - Nucleus 1-6: 481-486, 2010.

Dobley, J., Iltis, H.H.: Taxonomy of Zea (Gramineae). I. A subgeneric classification with key to taxa. - Amer. J. Bot. 67: 982-993, 1980.

Dover, G.A., Riley, R.: The effect of spindle inhibitors applied before meiosis on meiotic chromosome pairing. - J. Cell. Sci. 12: 143-161, 1973.

Driscoll, C.J., Darvey, N.L.: Chromosome pairing: effect of colchicine on an isochromosome. - Sci. 169: 290-291, 1970.

Driscoll, C.J., Darvey, N.L., Barber, H.N.: Effect of colchicine on meiosis of hexaploid wheat. - Nature 216: 687-688, 1967.

Feldman, M., Avivi, L.: Kew Chromosome Conference III - In Brandham PE (ed.) Genetic control of bivalent pairing in common wheat. The mode of Ph1 action. Pp.269-279. - Kew Conference III - London, 1988. 
Feldman, F., Liu, B., Segal, G., Abbo, S., Levy. A.: Rapid elimination of low copy DNA sequences in polyploidy wheat: a possible mechanism for differentiation of homeologous chromosomes. - Genet. 147: 1381-1387, 1997.

Fukunaga, K., Hill, J., Vigoroux, Y., Matsuoka, Y., Sanchez G., J., Liu, K., Bucker, E., Doebley, J.: Genetic diversity and population structure of Teosinte. - Genet. 169: 2241-2254.

Furini, A., Jewell, C.: Somatic embryogenesis and plant regeneration of maize/Tripsacum hybrids. - Maydica 40(2) :205-210, 1995.

Goluboskaya, I.N., Harper, L.C., Pawlowski, W.P., Schicnes, D.; Cande, W.Z.: The pam1 gene is required for meiotic bouquet formation and efficient homologous synapsis in maize (Zea mays L.). - Genet. 162: 1979-1993, 2002.

García, M.D., Molina, M. del C.: Embryo rescue and induction of somatic embryogenesis as a method to overcome seed inviability in Zea mays ssp. mays $(2 \mathrm{n}=40) \times$ Zea mays ssp. parviglumis crosses. - Biol. Plant. 44: 497-501, 2001.

García, M.D., Molina, M. del C., Caso, 0.H.: La regeneración de plantas de maíz (Zea mays ssp. mays) a partir del cultivo de tejidos y sus aplicaciones en el mejoramiento de maíz. - Rev. de la Fac. de Agronomía de la UNLP 68: 15-25, 1992. [In Spanish]

González, G., Poggio, L.: Karyotype of Zea luxurians and Z. mays subsp. mays using FISH/DAPI, and analysis of meiotic behavior of hybrids. Genome 54: 26-32, 2011.

Harper, L., Golubovskaya, I., Cande W.Z.: A bouquet of chromosomes. - J. of Cell Sci. 117: 4025-4032, 2004.

Iltis, H.H., Benz B.F: Zea nicaraguensis (Poaceae), a new teosinte from Pacific coastal Nicaragua. - Novon 10-4: 382-390, 2000.

Iltis, H.H.; Dobley J.: Taxonomy of Zea (Gramineae). II Subspecific categories in the Zea mays complex and a generic synopsis. - Amer. J. Bot. 67: 994-1004, 1980.

Jackson, R.C.: Polyploidy and diploidy: New perspectives on chromosome pairing and its evolutionary implications. - Amer. J. Bot. 69:1512-1523, 1982.

Jackson, R.C., Murray, B.G.: Colchicine-induced quadrivalent formation in Helianthus: evidence of ancient polyploidy. - Theor. Appl. Genet. 64: 219-222, 1983.

Jenczewski, E., Alix, K.: From diploids to allopolyploids: the emergence of efficient pairing control genes in plants. - Crit. Rev. Plant Sci. 23: 21-25, 2004.

Jenkins, G., Chatterjee, R.: Chromosome structure and pairing preferences in tetraploid rye (Secale cereale). - Genome 37: 784-793, 1994.

Molina, M. DEL C.: Estudios citogenéticos evolutivos del Género Zea. - Doctoral thesis at the Polytechnic University of Valencia, Spain, 2011. [In Spanish].

Molina, M. DEL C., Chorzempa, S.E.; García, M.D.: Meiotic pairing in the hybrid (Zea mays $x$ Zea diploperennis) x Zea luxurians. - Maize Genet. Coop. Newsletter 79: 5-7, 2005. 
Molina, M. DEL C., García, M.D.: Influence of ploidy levels on phenotypic and cytogenetic traits in maize and Zea perennis hybrids. - Cytologia 64: 101-109, 1999.

Molina, M. DEL C., García, M.D.: Meiotic pairing in the interspecific hybrid Zea mays, Z. perennis and Zea diploperennis. - Maize Genet. Coop. Newsletter 74: 42-43, 2000.

Molina, M. DEL C., García, M.D.: Ploidy levels affect phenotype and cytogenetic traits in Zea mays ssp. mays ( $2 \mathrm{n}=20$ or 40$)$ and Zea mays ssp. parviglumis hybrids. - Cytologia 66-2: 189-196, 2001.

Molina, M. DEL C., Naranjo, C.A.: Cytogenetic studies in the genus Zea. I. Evidence for five as the basic chromosomes number. - Theor. Appl. Genet. 73: 542-550, 1987.

Molina, M. DEL C., García, M.D., López C.G., Moreno Ferrero, V.: Meiotic pairing in the hybrid (Zea diploperennis x Zea perennis) x Zea mays and its reciprocal. - Hereditas 141: 1-7, 2004.

Naranjo, C.A., Molina, M.C., Poggio, L.: Evidencias de un número básico $x=5$ en el género Zea y su importancia en estudios del origen del maíz. - Acad. Nac. Cs. Ex. Fis. y Nat. 5: 75-84, 1989. [In Spanish].

Naranjo, C.A., Poggio, L., Molina, M.C., Bernatene, E.: Increase in multivalent frequency in F1 hybrids of Zea diploperennis X Z. perennis by colchicine treatment. - Hereditas 120: 241244, 1994.

Poggio, L., Molina, M.C., Naranjo, C.A.: Cytogenetic studies in the genus Zea. 2- colchicineinduced multivalents. -Theor. Appl. Genet. 79: 461-464, 1990.

Ruiz, C., Sanchez, J.J., Aguilar, S.M.: Potential geographical distribution of teosinte in Mexico: a gish approach. - Maydica 46: 105-110, 2001.

Santos, J.L., Lacadena, J.R., Cermeno, M.C., Orellana, J.: Nucleolar organizer activity in Wheat-Barley chromosome addition lines. - Heredity 53-3: 425-429, 1984.

Santos, J.L., Orellana, J.: Pairing competition between identical and homologous chromosome in rye and grasshoppers. - Genet. 104: 677-684, 1983.

Schnable, J.C., Springer, N.M., Freeling, M.: Differentiation of maize subgenome by genome dominance and both ancient and ongoing gene loss. - PNAS 108-10: 4069-4074, 2011.

Schnable, J.C., Freeling, M.: Genes identified by visible mutant phenotypes show increased bias toward one of two subgenomes of maize. - PLoS ONE 6-3: e17855. Doi:101371/journal.pone.0017855, 2011.

Sokal, R.R., Rohlf, Y.: Biometría. - W.H. Freeman and Company. San Francisco, 1978.

Swanson-Wagner R., Eichten S., Kumari S., Tiffin P., Stein J., Ware D., Springer N.: Pervasive gene content variation and copy number variation in maize and its undomesticated progenitor. - Genome Research,

- http://www.genome.org/cgi/doi/10.1101/gr.109165.110., 2012. 
Swigonavá, Z., Lai, J., Ma, J., Ramakrisma, W., Llaca, V., Bennetzen, J., Messing, J.: Close split of sorghum and maize genome progenitors, - Genome Res. 14: 1916-1923, 2004 Wendel, J.: Genome evolution in polyploidy, - Plant Mol. Biol. 42: 225-229, 2000

Zickler, D., Kleckner, N.: The leptotene-zygotene transition of meiosis. - Annu. Rev. Genet. 32: 619-697, 1998. 


\begin{tabular}{|c|c|c|c|c|c|c|c|}
\hline \multirow{2}{*}{ Species } & \multicolumn{6}{|c|}{ IV/Cell } & \multirow{2}{*}{$\begin{array}{c}\text { № of cells } \\
\text { studied }\end{array}$} \\
\hline & 0 & 1 & 2 & 3 & 4 & 5 & \\
\hline \multicolumn{8}{|c|}{ A - Zea mays } \\
\hline Control & 100.0 & 0.0 & 0.0 & 0.0 & 0.0 & 0.0 & 200 \\
\hline Treated & 38.3 & 17.8 & 15.3 & 8.2 & 16.8 & 3.6 & 196 \\
\hline \multicolumn{8}{|c|}{ B - Zea mexicana } \\
\hline Control & 100.0 & 0.0 & 0.0 & 0.0 & 0.0 & 0.0 & 136 \\
\hline Treated & 37.7 & 25.7 & 18.0 & 7.8 & 6.6 & 4.2 & 167 \\
\hline \multicolumn{8}{|c|}{ C - Zea parviglumis } \\
\hline Control & 100.0 & 0.0 & 0.0 & 0.0 & 0.0 & 0.0 & 181 \\
\hline Treated & 34.3 & 20.0 & 17.8 & 12.0 & 13.1 & 2.8 & 175 \\
\hline \multicolumn{8}{|c|}{ D - Zea luxurians } \\
\hline Control & 100.0 & 0.0 & 0.0 & 0.0 & 0.0 & 0.0 & 162 \\
\hline Treated & 36.5 & 23.0 & 19.6 & 10.1 & 6.1 & 4.7 & 148 \\
\hline \multicolumn{8}{|c|}{ E - Zea diploperennis } \\
\hline Control & 100.0 & 0.0 & 0.0 & 0.0 & 0.0 & 0.0 & 164 \\
\hline Treated & 100.0 & 0.0 & 0.0 & 0.0 & 0.0 & 0.0 & 196 \\
\hline
\end{tabular}

Table 1: Percentage of quadrivalents in parental species with $2 n=20$, for plants treated with colchicine $\left(0.5 \times 10^{-4} \mathrm{M}\right)$ and untreated control plants (significance in parenthesis) : A-Zea mays ( $\mathrm{p}$-value 0.00$) ; B$ - Zea mexicana ( $\mathrm{p}$ value 0.00 ); $C$ - Zea parviglumis ( $p$-value 0.00 ); $D$ - Zea luxurians ( $p$-value 0.00 ); E- Zea diploperennis (p-value 1.00).

\begin{tabular}{llllllllll}
\hline & \multicolumn{1}{c}{} & \multicolumn{1}{c}{ IV/Cell } & & $\begin{array}{c}\text { № of cells } \\
\text { studied }\end{array}$ \\
\cline { 2 - 8 } & 0 & 1 & 2 & 3 & 4 & 5 & \\
\hline
\end{tabular}




\begin{tabular}{|c|c|c|c|c|c|c|c|}
\hline \multicolumn{8}{|c|}{ A - ZmxZmex } \\
\hline Control & 100.0 & 0.0 & 0.0 & 0.0 & 0.0 & 0.0 & 127 \\
\hline Treated & 35.2 & 18.6 & 22.8 & 3.4 & 9.7 & 10.3 & 145 \\
\hline \multicolumn{8}{|c|}{ B - ZmxZpar } \\
\hline Control & 100.0 & 0.0 & 0.0 & 0.0 & 0.0 & 0.0 & 177 \\
\hline Treated & 31.1 & 36.7 & 9.4 & 8.3 & 8.9 & 5.6 & 180 \\
\hline \multicolumn{8}{|l|}{$C-Z m \times Z I$} \\
\hline Control & 100.0 & 0.0 & 0.0 & 0.0 & 0.0 & 0.0 & 200 \\
\hline Treated & 59.0 & 15.4 & 13.4 & 4.4 & 4.9 & 2.9 & 203 \\
\hline \multicolumn{8}{|l|}{$D-Z m x Z d$} \\
\hline Control & 100.0 & 0.0 & 0.0 & 0.0 & 0.0 & 0.0 & 181 \\
\hline Treated & 100.0 & 0.0 & 0.0 & 0.0 & 0.0 & 0.0 & 179 \\
\hline \multicolumn{8}{|l|}{$E-Z d x Z I$} \\
\hline Control & 100.0 & 0.0 & 0.0 & 0.0 & 0.0 & 0.0 & 154 \\
\hline Treated & 100.0 & 0.0 & 0.0 & 0.0 & 0.0 & 0.0 & 157 \\
\hline \multicolumn{8}{|l|}{ F - MDL } \\
\hline Control & 100.0 & 0.0 & 0.0 & 0.0 & 0.0 & 0.0 & 166 \\
\hline Treated & 62.0 & 8.8 & 11.1 & 9.9 & 5.3 & 2.9 & 171 \\
\hline
\end{tabular}

Table 2: Percentage of quadrivalents in hybrids with $2 n=20$, for plants treated with colchicine $\left(0.5 \times 10^{-4} \mathrm{M}\right)$ and untreated control plants (significance in parenthesis): A- Zea mays $\times$ Zea mexicana ( $\mathrm{p}$-value 0.00$)$; $B$ - Zea mays $\times$ Zea parviglumis with $2 \mathrm{n}=20$ ( $\mathrm{p}$-value 0.00); $C$ - Zea mays $\times$ Zea luxurians ( $\mathrm{p}$-value $\left.1.25 \times 10^{-13}\right) ; D$ - Zea mays $\times$ Zea diploperennis with $2 n=20$ ( $p$-value 1.00); $E$ Zea diploperennis x Zea luxurians ( $p$-value 1.00); F- Trihybrid between (Zea mays $x$ Zea diploperennis) $\times$ Zea luxurians ( $\mathrm{p}$-value $\left.1.60 \times 10^{-11}\right)$.

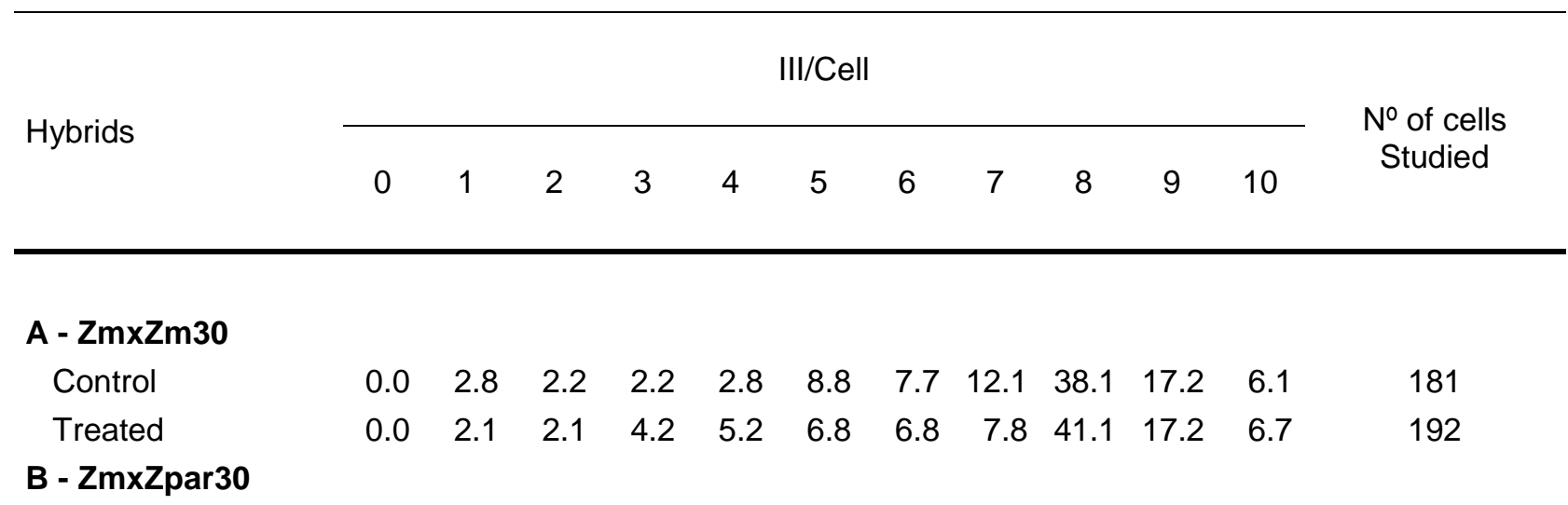




$\begin{array}{lrrrrrrrrrrrr}\text { Control } & 5.1 & 7.2 & 11.6 & 13.8 & 19.6 & 29.0 & 5.7 & 3.6 & 2.2 & 0.0 & 2.2 & 138 \\ \quad \text { Treated } & 0.0 & 1.9 & 7.1 & 7.7 & 14.7 & 32.1 & 12.8 & 11.5 & 6.4 & 3.8 & 2.0 & 156 \\ \text { C - ZmxZp30 } & & & & & & & & & & & & \\ \quad \text { Control } & 0.0 & 0.0 & 0.0 & 2.8 & 30.0 & 59.3 & 5.7 & 2.2 & 0.0 & 0.0 & 0.0 & 140 \\ \quad \begin{array}{l}\text { Treated } \\ \text { D - ZpxZmex }\end{array} & 0.0 & 0.0 & 0.0 & 0.0 & 4.7 & 5.3 & 12.4 & 20.6 & 29.4 & 15.3 & 12.3 & 170 \\ \quad \text { Control } & & & & & & & & & & & & \\ \quad \text { Treated } & 0.0 & 0.0 & 3.8 & 5.0 & 26.1 & 58.4 & 6.7 & 0.0 & 0.0 & 0.0 & 0.0 & 180 \\ \text { E - ZlxZp30 } & 0.0 & 0.0 & 0.0 & 2.9 & 13.8 & 56.3 & 6.9 & 6.3 & 5.2 & 5.2 & 3.4 & 174 \\ \quad \text { Control } & & & & & & & & & & & & \\ \quad \text { Treated } & 3.1 & 4.4 & 8.1 & 6.9 & 23.1 & 26.2 & 11.9 & 8.1 & 5.0 & 1.9 & 1.3 & 160 \\ \text { F - ZdxZp30 } & 0.0 & 0.0 & 3.9 & 5.1 & 10.0 & 22.2 & 21.1 & 18.8 & 11.1 & 5.0 & 2.8 & 180 \\ \quad \text { Control } & & & & & & & & & & & & \\ \quad \text { Treated } & 0.0 & 0.0 & 4.2 & 4.8 & 18.1 & 69.9 & 3.0 & 0.0 & 0.0 & 0.0 & 0.0 & 166 \\ & 0.0 & 0.0 & 0.0 & 0.0 & 9.1 & 44.0 & 21.2 & 8.0 & 5.7 & 6.9 & 5.1 & 175\end{array}$

Table 3: Percentage of trivalents in parental species with $2 n=30$, for plants treated colchicine $\left(0.5 \times 10^{-4} \mathrm{M}\right)$ and untreated control plants (significance in parenthesis): $A$ - Hybrid between maize species with different ploidy level ( $p$ value 0.77$)$; $B$ - Zea mays $\times$ Zea parviglumis with $2 \mathrm{n}=30\left(\mathrm{p}\right.$-value $\left.1.13 \times 10^{-8}\right)$; $C$ - Zea mays $x$ Zea perennis with $2 \mathrm{n}=30$ ( $\mathrm{p}$-value 0.00$) ; D$ - Zea perennis $\mathrm{x}$ Zea Mexicana (p-value $\left.1.53 \times 10^{-8}\right)$; E- Zea luxurians $\times$ Zea perennis ( $\mathrm{p}$-value $\left.1.42 \times 10^{-10}\right) ; F$ - Zea diploperennis $\times$ Zea perennis with $2 \mathrm{n}=30$ ( $\mathrm{p}$-value $0.40 \mathrm{x}$ $\left.10^{-16}\right)$.

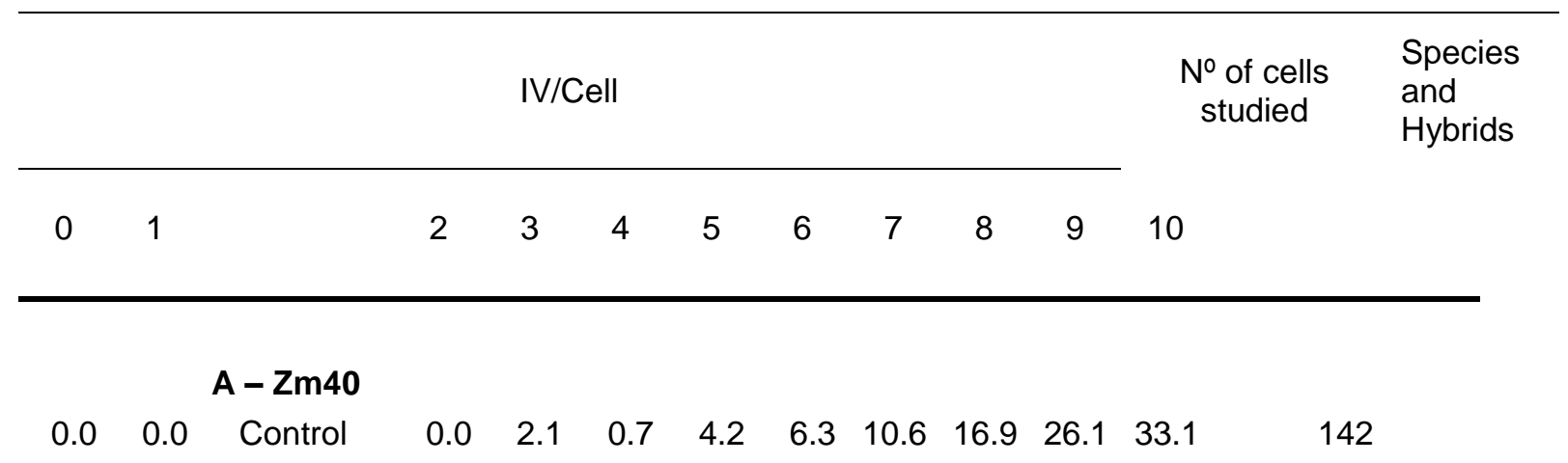




\begin{tabular}{|c|c|c|c|c|c|c|c|c|c|c|c|c|}
\hline 0.0 & 0.0 & Treated & 0.0 & 0.0 & 2.2 & 3.9 & 5.1 & 7.9 & 16.8 & 27.0 & 37.1 & $17 \varepsilon$ \\
\hline & & $B-Z p$ & & & & & & & & & & \\
\hline 0.0 & 2.3 & Control & 2.8 & 6.7 & 28.1 & 52.8 & 7.3 & 0.0 & 0.0 & 0.0 & 0.0 & $17 \varepsilon$ \\
\hline 0.0 & 0.0 & $\begin{array}{l}\text { Treated } \\
\text { C - } \\
\text { ZmxZp40 }\end{array}$ & 2.0 & 3.9 & 10.7 & 19.5 & 31.7 & 18.5 & 9.9 & 1.9 & 1.9 & 205 \\
\hline 3.1 & 5.8 & Control & 9.4 & 20.6 & 21.4 & 34.8 & 4.9 & 0.0 & 0.0 & 0.0 & 0.0 & 224 \\
\hline 0.0 & 0.0 & $\begin{array}{l}\text { Treated } \\
\text { D - } \\
\text { ZdxZp40 }\end{array}$ & 5.0 & 21.0 & 26.0 & 32.0 & 5.0 & 4.0 & 4.0 & 2.0 & 1.0 & 200 \\
\hline 1.9 & 2.8 & Control & 6.1 & 19.2 & 26.6 & 31.8 & 9.8 & 1.8 & 0.0 & 0.0 & 0.0 & 214 \\
\hline 0.0 & 0.0 & $\begin{array}{l}\text { Treated } \\
\text { E- } \\
\text { ZparxZd40 }\end{array}$ & 2.0 & 4.9 & 5.9 & 27.9 & 26.3 & 15.3 & 10.8 & 3.9 & 3.0 & 204 \\
\hline 15.1 & 26.9 & Control & 29 & 17.7 & 11.3 & 0.0 & 0.0 & 0.0 & 0.0 & 0.0 & 0.0 & 186 \\
\hline 0.0 & 7.8 & Treated & 16.7 & 18.9 & 15.6 & 11.1 & 13.9 & 5.0 & 3.3 & 4.4 & 3.3 & 180 \\
\hline & & F - MDP & & & & & & & & & & \\
\hline 1.2 & 8.0 & Control & 6.2 & 11.1 & 11.7 & 46.9 & 13.0 & 1.9 & 0.0 & 0.0 & 0.0 & 162 \\
\hline 0.0 & 0.0 & Treated & 1.9 & 5.0 & 6.9 & 30.0 & 28.1 & 11.2 & 8.1 & 5.0 & 3.8 & 160 \\
\hline
\end{tabular}

Table 4: Percentage of quadrivalents in parental species and Zea hybrids with $2 n=40$, for plants treated with colchicine $\left(0.5 \times 10^{-4} \mathrm{M}\right)$ and untreated control plants (significance in parenthesis): $A$ - Zea mays with $2 n=40$ ( $p$-value 0.33 ); $B$ - Zea perennis ( $\mathrm{p}$-value 0.00); C- Zea mays $x$ Zea perennis with $2 \mathrm{n}=40(\mathrm{p}$ value $\left.2.50 \times 10^{-5}\right) ; D$ - Zea diploperennis $\times$ Zea perennis with $2 \mathrm{n}=40(\mathrm{p}$-value 0.00); E- Zea parviglumis $x$ Zea diploperennis with $2 n=40$ ( $p$-value 0.00); Ftrihybrid between Zea mays $\times$ (Zea diploperennis $\times$ Zea perennis) with $2 n=40$ (p-value $\left.1.10 \times 10^{-16}\right)$.

\begin{tabular}{|c|c|c|c|}
\hline \multirow{2}{*}{$\begin{array}{c}\text { Species and } \\
\text { Hybrids }\end{array}$} & \multirow{2}{*}{$2 n$} & \multicolumn{2}{|c|}{ Chiasmas } \\
\hline & & Control & Treated \\
\hline $\mathrm{Zm}$ & 20 & 14.00 & 20.00 \\
\hline Zmex & 20 & 16.48 & 21.70 \\
\hline
\end{tabular}




\begin{tabular}{llll} 
Zpar & 20 & 15.36 & 20.08 \\
Zl & 20 & 15.34 & 21.34 \\
Zd & 20 & 14.00 & 14.64 \\
Zm x Zmex & 20 & 16.75 & 22.72 \\
Zm x Zpar20 & 20 & 15.42 & 21.06 \\
Zm x Zl & 20 & 15.48 & 20.36 \\
Zm x Zd20 & 20 & 15.00 & 15.35 \\
Zd x Zl & 20 & 15.26 & 16.24 \\
MDL & 20 & 14.57 & 19.38 \\
Zm x Zm30 & 30 & 28.12 & 29.03 \\
Zm x Zpar30 & 30 & 25.60 & 36.70 \\
Zm x Zp30 & 30 & 23.06 & 37.09 \\
Zp x Zmex & 30 & 24.02 & 32.50 \\
Zl x Zp & 30 & 21.36 & 29.60 \\
Zd x Zp30 & 30 & 17.25 & 26.34 \\
Zm40 & 40 & 33.75 & 34.25 \\
Zp & 40 & 34.56 & 41.80 \\
Zm x Zp40 & 40 & 33.59 & 42.50 \\
Zd x Zp40 & 40 & 31.42 & 40.36 \\
Zpar x Zd40 & 40 & 28.20 & 37.25 \\
MDP & 40 & 33.81 & 42.25 \\
\hline
\end{tabular}

Table 5: Average number of chiasmata in species and hybrids of Zea in material treated with a diluted colchicine solution and untreated control 


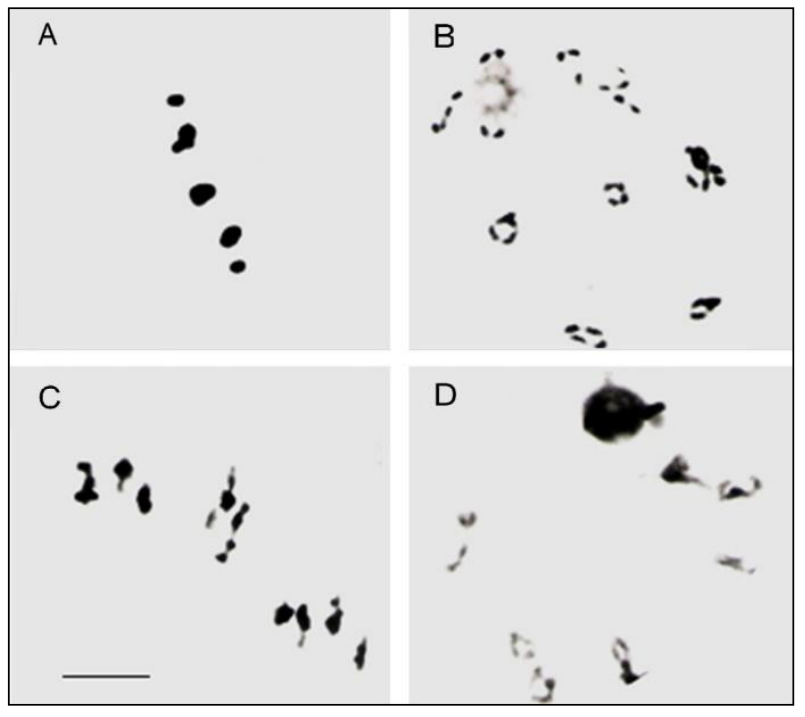

Fig. 1. Meiotic configurations induced by colchicine treatment in: $A-Z$. mays with $2 \mathrm{n}=$ 20 (5IV); B- Zea perennis (81V + 4II); C- Z. mays $\times$ Zea perennis with $2 \mathrm{n}=30 \quad$ (10III); $D$ - Z. mays $\times$ Zea perennis with $2 \mathrm{n}=40(9 \mathrm{IV}+2 \mathrm{II})$. Scale $10 \mu \mathrm{m}$. 
First evolutionary differenciation

AAAA $\longrightarrow$ AA BB

\section{Second evolutionary differenciation}

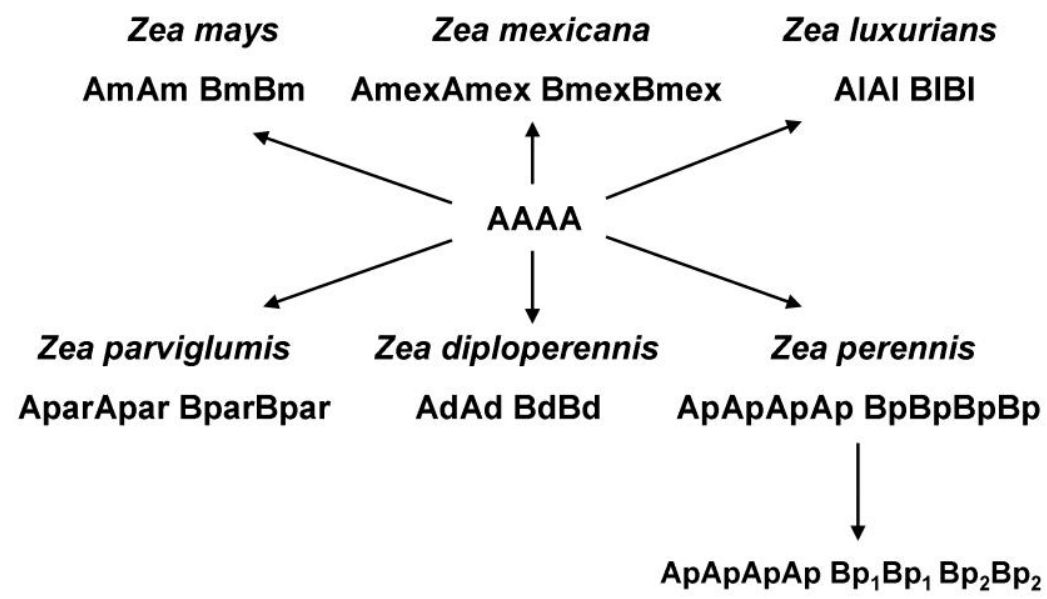

Fig. 2: Possible mechanisms of chromosome differentiation in the genus Zea. First differentiation: Tetraploid species with A genome would have differentiated into A and B genomes. Second differentiation: B genomes would have mutated or differentiated between them, resulting in different Zea species with $2 n=20$. Particularly in the case of Zea perennis, chromosomes of the B genome could have undergone further differentiation, which resulted in $\mathrm{Bp}_{1}$ and $\mathrm{Bp}_{2}$ homoeologous chromosomes. 